\title{
A Bertrand Duopoly Game with Long-Memory Effects
}

\author{
Baogui Xin $\mathbb{D},{ }^{1}$ Fangrui Cao, ${ }^{1}$ Wei Peng $\mathbb{D}^{1},{ }^{1}$ and Abdelalim A. Elsadany $\mathbb{D}^{2,3}$ \\ ${ }^{1}$ School of Economics and Management, Shandong University of Science and Technology, Qingdao 266590, China \\ ${ }^{2}$ Department of Mathematics, College of Sciences and Humanities Studies in Al-Kharj, Prince Sattam Bin Abdulaziz University, \\ Al-Kharj 11942, Saudi Arabia \\ ${ }^{3}$ Department of Basic Science, Faculty of Computers and Informatics, Suez Canal University, Ismailia 41522, Egypt
}

Correspondence should be addressed to Baogui Xin; xin@sdust.edu.cn

Received 29 April 2020; Accepted 30 May 2020; Published 29 July 2020

Guest Editor: Wei Zhou

Copyright (c) 2020 Baogui Xin et al. This is an open access article distributed under the Creative Commons Attribution License, which permits unrestricted use, distribution, and reproduction in any medium, provided the original work is properly cited.

Reconsidering the Bertrand duopoly game based on the concept of long short-term memory, we construct a fractional-order Bertrand duopoly game by extending the integer-order game to its corresponding fractional-order form. We build such a Bertrand duopoly game, in which both players can make their decisions with long-memory effects. Then, we investigate its Nash equilibria, local stability, and numerical solutions. Using the bifurcation diagram, the phase portrait, time series, and the 0 - 1 test for chaos, we numerically validate these results and illustrate its complex phenomena, such as bifurcation and chaos.

\section{Introduction}

As we know, the game theory is one of hottest academic topics for a long time. There are many types of games, such as the Cournot game [1-7], the Bertrand game [8-14], the Stackelberg game [15-17], the evolutionary game [18], and the mixed game [19]. Many researchers have studied duopoly games with some interesting characteristics, such as bounded rationality $[1,15-17]$, technology licensing $[3,20]$, and $\mathrm{R} \& \mathrm{D}[6,13]$. Table 1 shows some recent research studies on duopoly games with integer-order difference equations. Generally speaking, the duopoly Bertrand game presents the optimal pricing strategies for two players. As a kind of pricing game, the Bertrand game is an alternative to the Cournot game. The duopoly Bertrand game describes two firms competitively providing a kind of homogeneous product, setting simultaneously prices rather than quantities, and reaching equilibria with the price equal to the marginal cost. In the industrial economics domain, long- or short-term memory effects are widespread with different forms. Classical dynamical Bertrand games mainly have short-term memory effects. The discrete-time dynamical Bertrand game represented by the integer-order difference equation can reveal the length of memory effect by its order number. For example, the first-order discrete dynamical game can only remember the decision-making behaviors during the previous period and the second-order discrete dynamical game can remember the decision-making behaviors during the previous two periods. Although the integer-order difference equation has a powerful expression ability and can represent its complexity evolution characteristics of the duopoly game, it is not very easy for the integer-order discrete-time dynamical Bertrand games to reflect long-term memory effects. Thus, it is meaningful to study the discrete-time dynamical Bertrand games with fractional-order calculus.

We can employ two forms of fractional-order calculus to represent long-term memory effects. The one is the continuous fractional-order calculus, which mainly corresponds to the integer-order differential calculus. The other is the discrete fractional-order calculus, which mainly corresponds to the integer-order difference calculus. Many scholars have utilized continuous fractional differential operators to represent memory effects in economics. As an extension of the integer-order difference equation, the fractional-order difference equation has the function of long-term memory effects, which can make up the deficiency of the integerorder difference equation. The fractional difference 
TABLE 1: Recent studies on dynamical duopoly games with integer-order difference equations.

\begin{tabular}{lccc}
\hline Study & Game type & Decision variables & Characteristics \\
\hline Ueda; Tu and Wang & Cournot game & Quantity & Bounded rationality \\
Hsu, et al. & Cournot game & Quantity & Technology licensing \\
Al-khedhairi & Cournot game & Quantity & Differentiated players \\
Fanti et al.; Gori and Sodini; Ahmed et al. & Bertrand game & Price & Product differentiation \\
Askar and Al-khedhairi & Bertrand game & Price & Limited information 12, 14] \\
Ma and Lou & Bertrand game & Price & Integer delay \\
Tu et al. & Bertrand game & Price & R\&D \\
Yang, et al. & Stackelberg game & Quantity & Bounded rationality \\
Peng and Lu & Stackelberg game & Quantity & Bounded rationality \\
Shi; Le and Sheng & Stackelberg game & Quantity & Bounded rationality \\
Ma et al. & Mixed game & Price, quantity & Heterogeneity \\
Wu & Differential game & price & Technology licensing \\
\hline
\end{tabular}

equation, named the discrete fractional equation, can also be regarded as the discrete form of a fractional differential equation. Without a doubt, the fractional difference calculus also has a vast application space in the dynamical game. Using the discrete fractional calculus, Xin et al. [21] studied a Cournot game and revealed the evolution mechanism of two firms' output decisions. Different from Ref. [21], this article will employ the discrete fractional-order differential calculus to propose a Bertrand game, which can reveal the evolution mechanism of two firms' price decisions.

The remainder of this paper is organized as follows. In Section 2, we propose a discrete fractional-order Bertrand duopoly game with long-term memory effects. In Section 3, we study the game's Nash equilibria and local stability. In Section 4, we numerically verify the results by using bifurcation diagrams, phase portraits, maximal Lyapunov exponents, and the $0-1$ test algorithm. This paper concludes in Section 5.

\section{Modelling}

In this following, we consider a simple Bertrand-type duopoly common market as mentioned in classic economics. Two firms, labelled by $i=1,2$, provide homogeneous products with perfect substitutes and decide their different product prices in the light of the same market rule. During period $t \in Z^{+}$, let $p_{i}(t)$ and $q_{i}(t)$ represent the product price and output of firm $i$, respectively. Following the classical linear inverse demand function, we can obtain the following relationship of $q_{i}$ and $p_{i}$ :

$$
q_{i}(t)=1-p_{i}(t)+b p_{j}(t), \quad i, j=1,2, \text { and } i \neq j,
$$

where $b$ is a positive constant and denotes a substitution effect between two products.

Let the marginal costs be linear, i.e., $C_{i}(t)=c q_{i}(t), \quad i=1,2$, where $c$ is a positive constant and represents the marginal cost. Then, the production profit of firm $i$ is

$$
\Pi_{i}\left(p_{i}(t), p_{j}(t)\right)=\left(p_{i}(t)-c\right) q_{i}(t), \quad i, j=1,2, \text { and } i \neq j .
$$

Thus, the marginal profit of firm $i$ with respect to $p_{i}$ is

$$
\begin{gathered}
\Theta(t)=\frac{\partial \Pi_{i}\left(p_{i}(t), p_{j}(t)\right)}{\partial p_{i}(t)}=1+c-2 p_{i}(t)+b p_{j}(t), \\
i, j=1,2 \text {, and } i \neq j .
\end{gathered}
$$

In the classical dynamical duopoly game, every firm naively think its rivals' price in period $t+1$ is equal to the same as in period $t$. Obviously, the kind of game has no longmemory effect. In the following, we will introduce the discrete fractional-order calculus to the classical game; then, both firms make their decisions under a novel dynamical adjustment mechanism with a long memory and local estimation of marginal profit.

Assume that the first firm has no complete knowledge of the market demand function and makes its price decision under a dynamical adjustment mechanism with the bounded rationality and a long-term estimation of marginal profit. If the long-term marginal profit is greater (less) than zero, the firm decides to raise (cut) its product price at next period. Thus, we describe the following adjustment process of the product price:

$$
\Delta^{\alpha} p_{1}(t)=a p_{1}(t+\alpha-1) \Theta(t+\alpha-1),
$$

where $a>0$ represents the first firm's price adjustment speed and $\alpha \in(0,1)$ represents fractional-order number, i.e., the long-term memory effect.

Assume that the second firm makes its decision under a dynamical adjustment mechanism with simple rationality and a long-term estimation of marginal profit. That is, its price decision mainly depends on two aspects. The one is its long-term optimal reaction function $p_{2}^{*}$, and the other is its long-term price $p_{2}$. Thus, we write the second firm's price adjustment mechanism as follows:

$$
\Delta^{\alpha} p_{2}(t)=p_{2}^{*}(t+\alpha-1)-p_{2}(t+\alpha-1)
$$

Thus, we write the following discrete-time fractionalorder Bertrand duopoly game with a long memory:

$$
\left\{\begin{array}{l}
\Delta^{\alpha} p_{1}(t)=a p_{1}(t+\alpha-1)\left(1+c-2 p_{1}(t+\alpha-1)+b p_{2}(t+\alpha-1)\right), \\
\Delta^{\alpha} p_{2}(t)=\frac{1}{2}\left(1+c+b p_{1}(t+\alpha-1)\right)-p_{2}(t+\alpha-1) .
\end{array}\right.
$$


Remark 1. When $\alpha=1$, equation (6) degenerates to the following equations:

$$
\left\{\begin{array}{l}
p_{1}(t+1)=p_{1}(t)+a p_{1}(t)\left(1+c-2 p_{1}(t)+b p_{2}(t)\right) \\
p_{2}(t+1)=\frac{1}{2}\left(1+c+b p_{1}(t)\right) .
\end{array}\right.
$$

\section{Nash Equilibrium and Local Stability}

To obtain equilibria of system (6), we solve the following equations:

$$
\left\{\begin{array}{l}
k p_{1}(t)\left(1+c-2 p_{1}(t)+b p_{2}(t)\right)=0 \\
\frac{1}{2}\left(1+c+b p_{1}\right)-p_{2}=0
\end{array}\right.
$$

Their two Nash equilibria are $E_{1}=(0,(1+c) / 2)$ and $E_{2}=\left((b c+b+2) /\left(4-b^{2}\right),(b+2 c+2) /\left(4-b^{2}\right)\right)$. In economics, their equilibria mean the folllowing:

(i) The equilibrium $E_{1}$ means that the best price of the first firm is $p_{1}^{*}=0$ if the second firm sets its optimal product price $p_{2}^{*}=(1+c) / 2$. Similarly, the best price of the second firm is $p_{2}^{*}=(1+c) / 2$ if the firm adopts zero price strategy. Obviously, $E_{1}$ is a bounded equilibrium [22].

(ii) The equilibrium $E_{2}$ means that both firms will maintain their equilibrium prices together because no firm can obtain any extra benefit by deviating unilaterally from its own equilibrium. Obviously, $E_{2}$ is a nonbounded equilibrium point.

Since the equilibrium $E_{1}$ has no real economic significance, we only analyze the complexity of the nonbounded equilibrium $E_{2}$.

The Jacobian matrix of system (6) computed at $E_{2}$ is

$$
J\left(E_{2}\right)=\left(\begin{array}{cc}
\frac{2 a(b c+b+2)}{b^{2}-4} & \frac{a b(b c+b+2)}{4-b^{2}} \\
\frac{b}{2} & -1
\end{array}\right) .
$$

From [21, 23], we can directly obtain Theorem 1 .

Theorem 1. System (5) is locally asymptotically stable at $E_{2}$ if $\operatorname{det} J>0$ and either

$$
\begin{array}{r}
\frac{-t r J}{2} \geq \sqrt{\operatorname{det} J} \text { and } \alpha>\log _{2} \frac{\sqrt{A}-t r J}{2}, \\
\frac{|t r J|}{2}<\sqrt{\operatorname{det} J}<\left(2 \cos \frac{B-\pi}{2-\alpha}\right)^{\alpha} \text { and } \alpha<\frac{2 B}{\pi},
\end{array}
$$

where $\operatorname{tr} J=\left(2 a b(1+c)+4 a-b^{2}+4\right) /\left(b^{2}-4\right), \operatorname{det} J=a / 2$ $(b c+b+2), A=\left|(\operatorname{tr} J)^{2}-4 \operatorname{det} J\right|$, and $B=\operatorname{tr} J / \sqrt{A}$.

\section{Numerical Simulation}

From [24, 25], we can directly obtain Theorem 2 .

Theorem 2. For the following nonlinear system,

$$
\left\{\begin{array}{l}
{ }^{C} \Delta_{0}^{\alpha} X(t)=F(t+\alpha-1, X(t+\alpha-1)), \\
\Delta^{j} X(0)=X_{j}, \quad j=0, \ldots, n-1, \quad n=[\alpha]+1 .
\end{array}\right.
$$

The equivalent form of system (12) is

$$
X(n)=X(0)+\frac{1}{\Gamma(\alpha)} \sum_{j=1}^{n} \frac{\Gamma(n-j+\alpha)}{\Gamma(n-j+1)} F(X(j-1)), \quad n \in \mathbb{N} .
$$
form:

So we can rewrite system (5) as the following numerical

$$
\left\{\begin{array}{l}
p_{1}(n)=p_{1}(0)+\frac{1}{\Gamma(\alpha)} \sum_{j=1}^{n} \frac{\Gamma(n-j+\alpha)}{\Gamma(n-j+1)} a p_{1}(j-1)\left(1+c-2 p_{1}(j-1)+b p_{2}(j-1)\right), \\
p_{2}(n)=p_{2}(0)+\frac{1}{\Gamma(\alpha)} \sum_{j=1}^{n} \frac{\Gamma(n-j+\alpha)}{\Gamma(n-j+1)} \frac{1}{2}\left(1+c+b p_{1}(j-1)\right)-p_{2}(j-1) .
\end{array}\right.
$$

Thus, we can illustrate the complexity of system (5) by using system (14). For equation (14), we set parameters $a=1.5, b=0.29, c=0.1, \alpha=0.999$, and the initial point $\left(p_{1}(0), p_{2}(0)\right)=(0.5,0.65)$. From Theorem 1 , we can obtain the following results: the nonbounded Nash equilibrium $E_{2}=(0.5922,0.6359)$, det $J=1.7393>0, \operatorname{tr} J=-2.7766<0$, $A=0.7525$, and $B=-3.2008$, so equation (10) holds. Thus, equation (5) is locally asymptotically stable at $E_{2}=(0.5922$, 0.6359 ). Figure 1 validates the results mentioned above.

In the following, we fix parameters $b=0.2$ and $c=0.5$, and the initial point $\left(p_{1}(0), p_{2}(0)\right)=(0.3,0.3)$ in system (14). We will study chaos by using the bifurcation diagram, the phase portrait, the maximal Lyapunov exponent, and 0-1 test algorithm for chaos [26-28]. To reveal the complexity of 


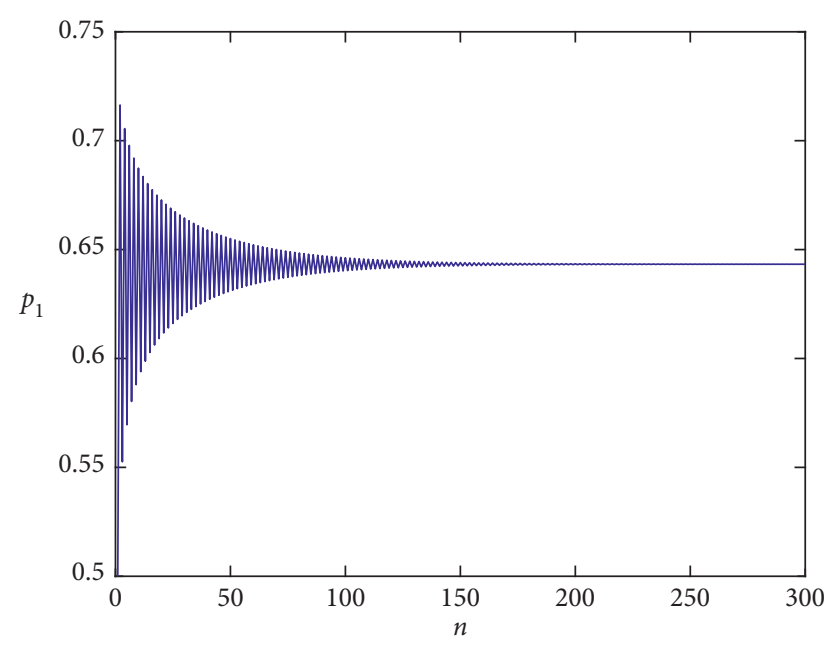

(a)

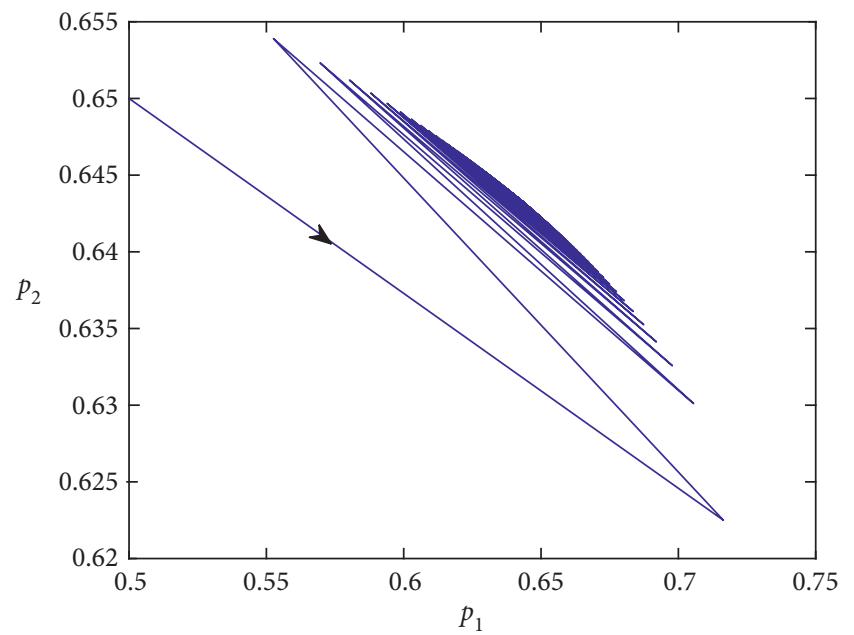

(b)

Figure 1: Stabilized states of duopoly pricing in system (14) with $\left(p_{1}(0), p_{2}(0)\right)=(0.5,0.65)$. (a) Price time series of firm 1 . (b) Price phase portrait of two firms.

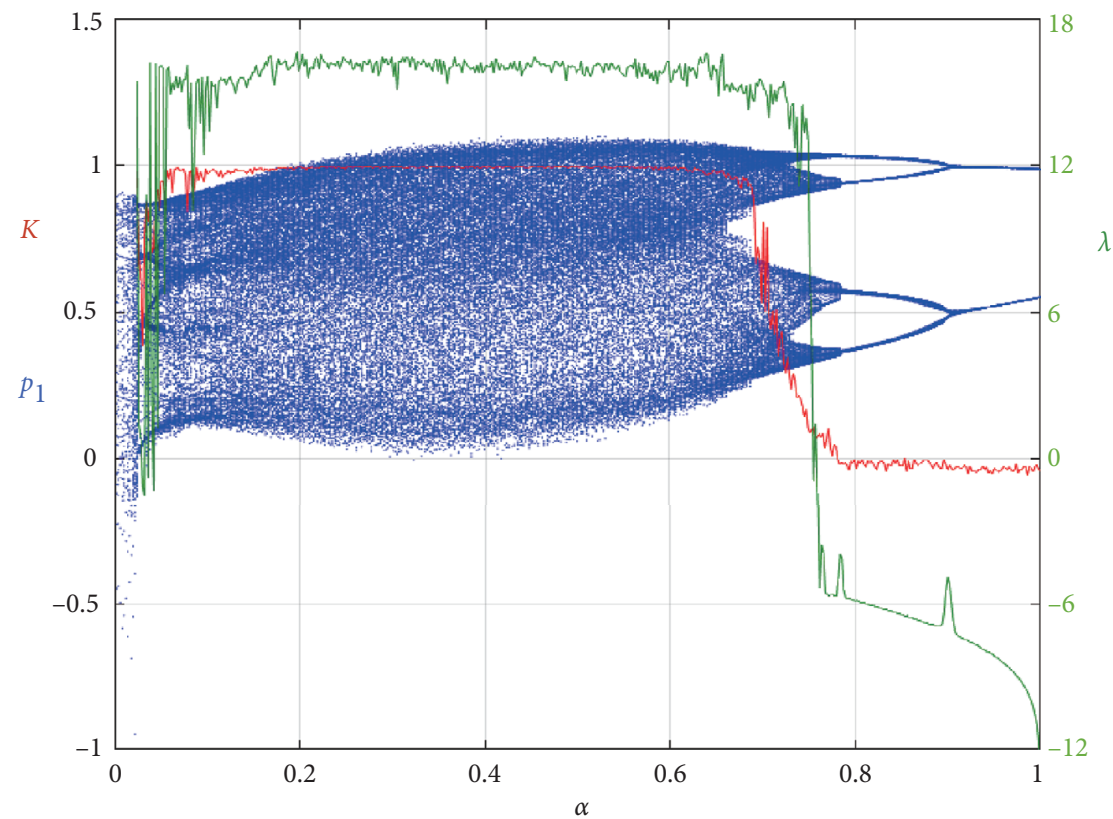

Figure 2: Firm 1's price diagrams of bifurcation ( $p_{1}$, blue), maximal Lyapunov exponent ( $\lambda$, dark green), and the median value of the correlation coefficient (K, red) in system (14) with varying $\alpha \in(0,1)$.

system (14), we will study the influence of two main parameters: long-term memory effect and price adjustment speed.

4.1. Complexity with Varying the Order $\alpha \in(0,1)$. To illustrate the complexity of system (14), such as bifurcation and chaos, we fix $a=1.4$ and vary the order $\alpha \in(0,1)$ with an increment of 0.002 . We integrate three subfigures to Figure 2. Figure 2 has the $y$-axis divided into left and right (for $K$ and $\lambda$ ) for better readability. The first subfigure is drawn for the bifurcation diagram of firm 1's price decision. The second subfigure is drawn for the median value $K$ of the correlation coefficient. The last subfigure is drawn for the maximal Lyapunov exponent $\lambda$ of firm 1's price decision. Figure 2 shows that the bifurcation diagram is in line with $K$ and $\lambda$.

At $\alpha=0.2$ in Figure 2 , we note that $K=0.9966 \approx 1$ and the maximal Lyapunov exponent $\lambda=16.1760>0$, which indicate there exists chaos in system (14). To confirm the results in Figure 2, we draw Figure 3 with the abovementioned parameter values. Figures $3(\mathrm{a})$ and $3(\mathrm{~b})$ show that the time series of firms 1 and 2 are nonperiodic. 


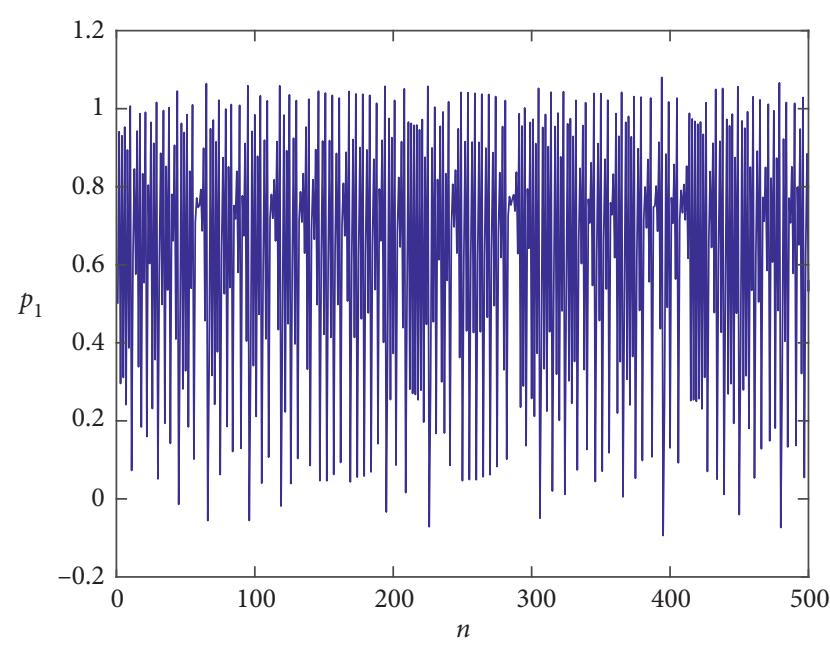

(a)

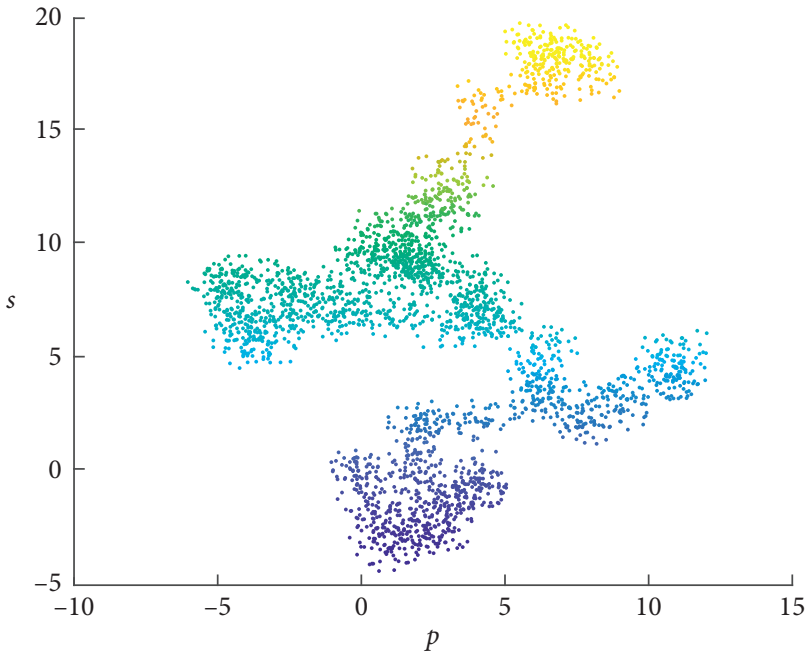

(c)

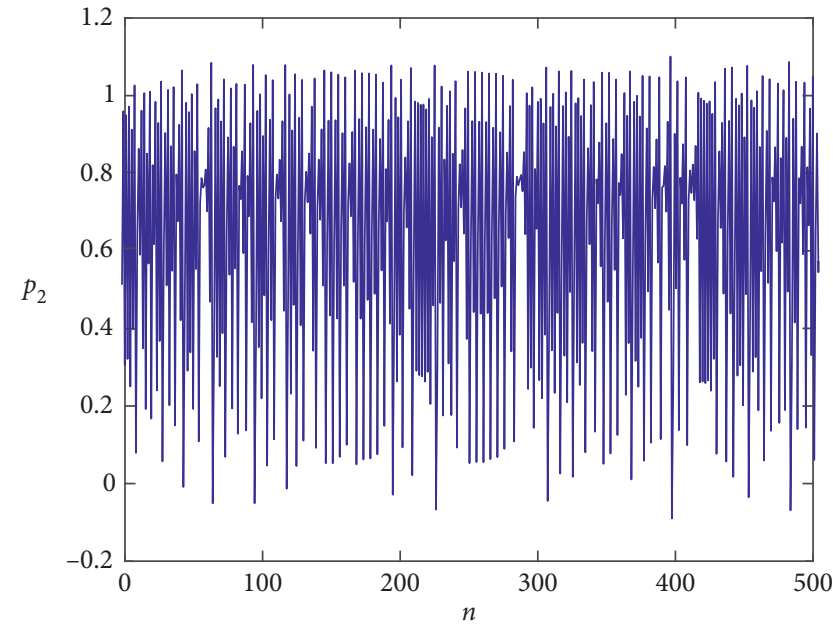

(b)

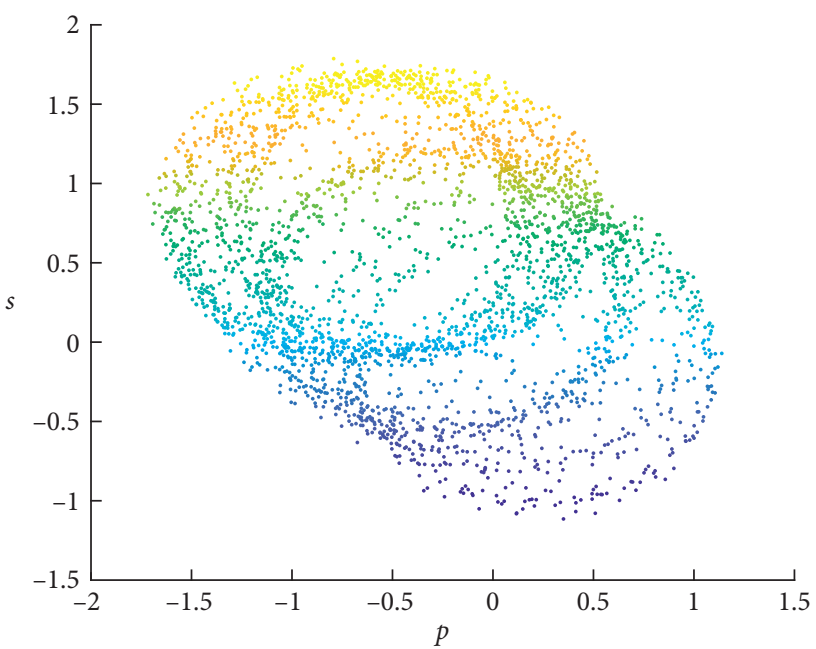

(d)

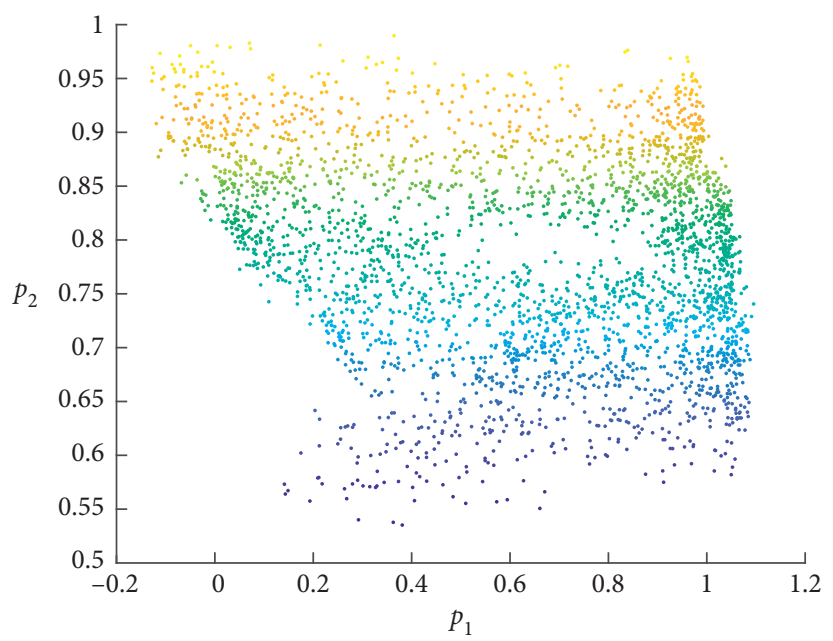

(e)

Figure 3: Chaos in system (14) with $\left(p_{1}(0), p_{2}(0)\right)=(0.3,0.3)$. (a) Price time series of firm 1. (b) Price time series of firm 2. (c) Firm 1's pricing translation components $(p, s)$. (d) Firm 2's pricing translation components $(p, s)$. (e) Phase portrait of duopoly pricing. 


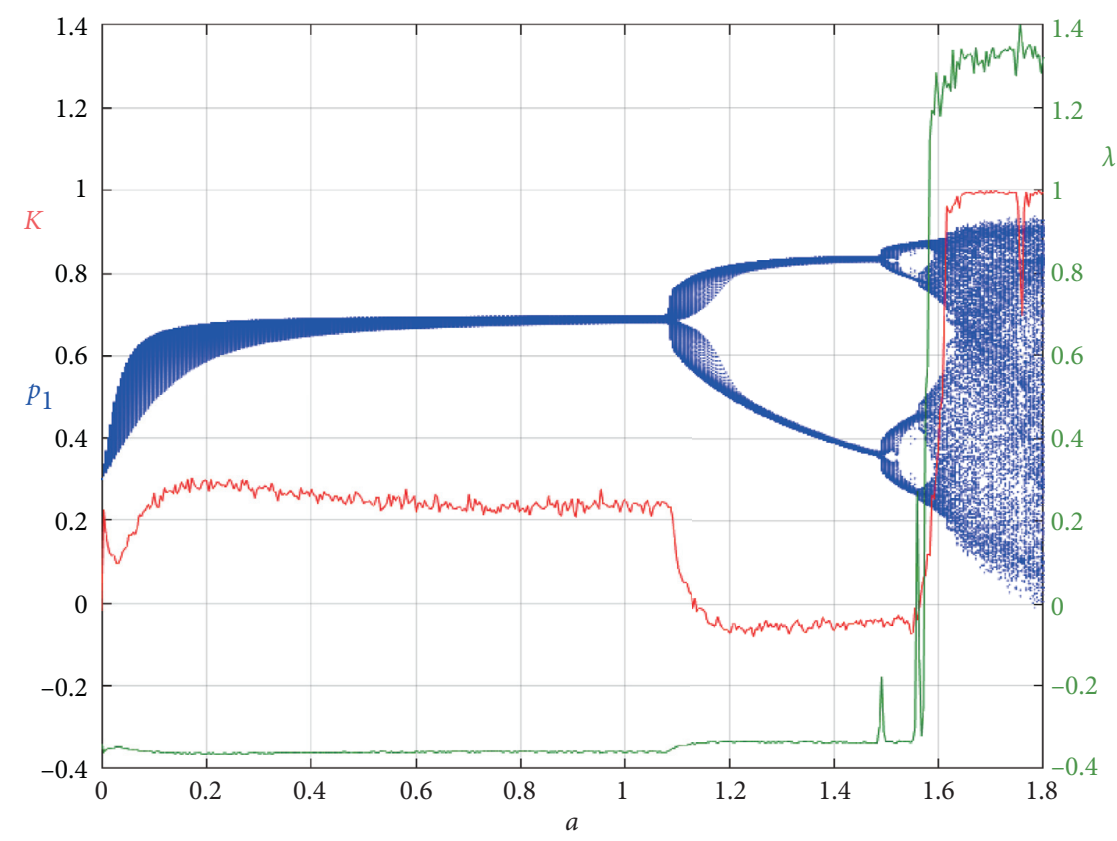

Figure 4: Firm 1's price diagrams of bifurcation ( $p_{1}$, blue), maximal Lyapunov exponent ( $\lambda$, dark green), and the median value of the correlation coefficient $(K$, red) in system (14) with varying $a \in(0,1.8)$.

Figures 3(c) and 3(d) demonstrate that the pricing trajectories of $K$ in the transformed coordinate $(p, s)$ of firms 1 and 2 are Brownian-like. Figure 3(e) illustrates that the phase portrait of duopoly pricing is chaotic. They quite well coincide with Figure 2.

4.2. Complexity with Varying the Price Adjustment Speed $a \in(0,1.8)$. To illustrate how the price adjustment speed $a$ affects the complexity of system (14), we use abovementioned parameter values, then set the order $\alpha=0.6$, and vary the price adjustment speed $a \in(0,1.8)$ with an increment of 0.004 . We also integrate three subfigures to Figure 4 . Figure 4 also has the $y$-axis divided into left and right (for $K$ and $\lambda$ ) for better readability. The three subfigures are the bifurcation diagram, the median value $K$ of the correlation coefficient, and the maximal Lyapunov exponent $\lambda$ of firm 1's price decision, respectively. Figure 4 also shows that the bifurcation diagram is in line with $K$ and $\lambda$.

The phase portrait and time series are similar to those in Section 4.1. For saving readers' time, we will not demonstrate the consistency between the phase portraits, the time series, the bifurcation diagram, the median value of the correlation coefficient, and the maximal Lyapunov exponent of firm 1's price decision.

\section{Conclusion and Future Developments}

Introducing the discrete fractional-order calculus into the integer-order nonlinear Bertrand game, we obtain a fractional-order Bertrand game with a long memory. Then, we qualitatively analyze the game's Nash equilibria and local stability. Finally, we validate these qualitative results and compare the consistency among bifurcation diagrams, phase portraits, time series, the maximal Lyapunov exponent, and the $0-1$ test values.

These results illustrate the complexity of the discrete fractional-order nonlinear Bertrand game. When the longterm memory effect increases from 0 to 1 , the game's complexity decreases gradually. When the price adjustment speed increases from 0 to 1.8 , the game's complexity increases gradually. So we can design suitable game mechanisms according to actual demand by changing the size of the long-term memory effect or the price adjustment speed.

Also, we can extend some researches in the following potential directions. First, there are many types of games that can be extended by discrete fractional-order calculus, such as the Edgeworth game or the Bertrand-Edgeworth game or the Cournot-Bertrand game. Second, we can study the control and synchronization of discrete fractional-order games. Third, we can study the stochastic or fuzzy versions of a discrete fractional-order game. Fourth, we can construct data-driven models of a discrete fractional-order game. At last, we can construct discrete fractional-order models in many scientific fields [29-33].

\section{Data Availability}

The data used to support the findings of this study are available from the corresponding author upon request.

\section{Conflicts of Interest}

The authors declare that they have no conflicts of interest.

\section{Acknowledgments}

This work was supported by the Natural Science Foundation of Shandong Province (Grant no. ZR2016FM26) and 
National Social Science Foundation of China (Grant no. 16FJY008).

\section{References}

[1] M. Ueda, "Effect of information asymmetry in Cournot duopoly game with bounded rationality," Applied Mathematics and Computation, vol. 362, no. 8, 2019.

[2] H. Tu and X. Wang, "Research on a dynamic master-slave Cournot triopoly game model with bounded rational rule and its control," Mathematical Problems in Engineering, vol. 2016, Article ID 2353909, 11 pages, 2016.

[3] J. Hsu, L. Liu, X. Henry Wang, and C. Zeng, "Ad valorem versus per-unit royalty licensing in a Cournot duopoly model," The Manchester School, vol. 87, no. 6, pp. 890-901, 2019.

[4] A. Al-khedhairi, "Differentiated Cournot duopoly game with fractional-order and its discretization," Engineering Computations, vol. 36, no. 3, pp. 781-806, 2019.

[5] B. Xin and Y. Wang, "Stability and Hopf bifurcation of a stochastic Cournot duopoly game in a blockchain cloud services market driven by brownian motion," IEEE Access, vol. 8, pp. 41432-41438, 2020.

[6] J. Zhou, W. Zhou, T. Chu, Y.-X. Chang, and M.-J. Huang, "Bifurcation, intermittent chaos and multi-stability in a twostage Cournot game with R\&D spillover and product differentiation," Applied Mathematics and Computation, vol. 341, pp. 358-378, 2019.

[7] Y.-h. Zhang, W. Zhou, T. Chu, Y.-D. Chu, and J.-N. Yu, "Complex dynamics analysis for a two-stage Cournot duopoly game of semi-collusion in production," Nonlinear Dynamics, vol. 91, no. 2, pp. 819-835, 2018.

[8] L. Gori and M. Sodini, "Price competition in a nonlinear differentiated duopoly," Chaos, Solitons \& Fractals, vol. 104, pp. 557-567, 2017.

[9] S. S. Askar and A. Al-khedhairi, "Dynamic investigations in a duopoly game with price competition based on relative profit and profit maximization," Journal of Computational and Applied Mathematics, vol. 367, Article ID 112464, 2020.

[10] B. Xin, W. Peng, and L. Guerrini, "A continuous time Bertrand duopoly game with fractional delay and conformable derivative: modelling, discretization process, Hopf bifurcation and chaos," Frontiers in Physics, vol. 7, p. 84, 2019.

[11] J. Ma and W. Lou, "Complex characteristics of multichannel household appliance supply chain with the price competition," Complexity, vol. 2017, Article ID 4327069, 12 pages, 2017.

[12] L. Fanti, L. Gori, C. Mammana, and E. Michetti, "The dynamics of a Bertrand duopoly with differentiated products: synchronization, intermittency and global dynamics," Chaos, Solitons \& Fractals, vol. 52, pp. 73-86, 2013.

[13] H. Tu, X. Zhan, and X. Mao, "Complex dynamics and chaos control on a kind of Bertrand duopoly game model considering R\&D activities," Discrete Dynamics in Nature and Society, vol. 2017, Article ID 7384150, 13 pages, 2017.

[14] E. Ahmed, A. A. Elsadany, and T. Puu, "On Bertrand duopoly game with differentiated goods," Applied Mathematics and Computation, vol. 251, pp. 169-179, 2015.

[15] X. Yang, Y. Peng, Y. Xiao, and X. Wu, "Nonlinear dynamics of a duopoly Stackelberg game with marginal costs," Chaos, Solitons \& Fractals, vol. 123, pp. 185-191, 2019.

[16] Y. Peng and Q. Lu, "Complex dynamics analysis for a duopoly Stackelberg game model with bounded rationality," Applied Mathematics and Computation, vol. 271, pp. 259-268, 2015.

[17] L. Shi, Y. Le, and Z. Sheng, "Analysis of price Stackelberg duopoly game with bounded rationality," Discrete Dynamics in Nature and Society, vol. 2014, Article ID 428568, 8 pages, 2014.

[18] Y. Dong, Y. Zhang, J. Pan, and T. Chen, "Evolutionary game model of stock price synchronicity from investor behavior," Discrete Dynamics in Nature and Society, vol. 2020, Article ID 7957282, 9 pages, 2020.

[19] J. Ma, L. Sun, S. Hou, and X. Zhan, "Complexity study on the Cournot-Bertrand mixed duopoly game model with market share preference," Chaos: An Interdisciplinary Journal of Nonlinear Science, vol. 28, no. 2, Article ID 023101, 2018.

[20] C.-H. Wu, "Licensing to a competitor and strategic royalty choice in a dynamic duopoly," European Journal of Operational Research, vol. 279, no. 3, pp. 840-853, 2019.

[21] B. Xin, W. Peng, and Y. Kwon, A Fractional-Order Difference Cournot Duopoly Game with Long Memory, Cornell University Press, Ithaca, NY, USA, 2019.

[22] G. I. Bischi and A. Naimzada, "Global analysis of a dynamic duopoly game with bounded rationality," in Advances in Dynamic Games and Applications, pp. 361-385, Springer, Birkhuser Boston, 2000.

[23] J. Cermak, I. Gyori, and L. Nechvatal, "On explicit stability conditions for a linear fractional difference system," Fractional Calculus and Applied Analysis, vol. 18, no. 3, pp. 651672, 2015.

[24] F. Chen, X. Luo, and Y. Zhou, "Existence results for nonlinear fractional difference equation," Advances in Difference Equations, vol. 2011, no. 1, pp. 1-12, 2011.

[25] G.-C. Wu and D. Baleanu, "Discrete fractional logistic map and its chaos," Nonlinear Dynamics, vol. 75 , no. 1-2, pp. 283-287, 2014.

[26] G. A. Gottwald and I. Melbourne, "On the validity of the 0-1 test for chaos," Nonlinearity, vol. 22, no. 6, pp. 1367-1382, 2009.

[27] I. Falconer, G. A. Gottwald, I. Melbourne, and K. Wormnes, "Application of the 0-1 test for chaos to experimental data," SIAM Journal on Applied Dynamical Systems, vol. 6, no. 2, pp. 395-402, 2007.

[28] L. Yuan, S. Zheng, and Z. Alam, "Dynamics analysis and cryptographic application of fractional logistic map," Nonlinear Dynamics, vol. 96, no. 1, pp. 615-636, 2019.

[29] T. Chen, B. Xiao, and H. Liu, "Credit risk contagion in an evolving network model integrating spillover effects and behavioral interventions," Complexity, vol. 2018, Article ID 1843792, 16 pages, 2018.

[30] T. Chen, J. Wang, H. Liu, and Y. He, "Contagion model on counterparty credit risk in the crt market by considering the heterogeneity of counterparties and preferential-random mixing attachment," Physica A: Statistical Mechanics and Its Applications, vol. 520, pp. 458-480, 2019.

[31] T. Chen, Y. Wang, Q. Zeng, and J. Luo, "Network model of credit risk contagion in the interbank market by considering bank runs and the fire sale of external assets," Physica A: Statistical Mechanics and Its Applications, vol. 542, Article ID 123006, 2020.

[32] B. Xin and M. Sun, "A differential oligopoly game for optimal production planning and water savings," European Journal of Operational Research, vol. 269, no. 1, pp. 206-217, 2018.

[33] B. Xin, W. Peng, Y. Kwon, and Y. Liu, "Modeling, discretization, and hyperchaos detection of conformable derivative approach to a financial system with market confidence and ethics risk," Advances in Difference Equations, vol. 2019, no. 1, p. 138, 2019. 